University of New Orleans

ScholarWorks@UNO

$2-2006$

\title{
Annealing effects on the crystallite size and dielectric properties of ultrafine Ba1-xSrxTiO3 $(0<x<1)$ powders synthesized through an oxalate-complex precursor
}

Gabriel Caruntu

University of New Orleans, gcaruntu@uno.edu

Charles J. O'Connor

University of New Orleans, coconnor@uno.edu

Follow this and additional works at: https://scholarworks.uno.edu/chem_facpubs

Part of the Chemistry Commons

\section{Recommended Citation}

Caruntu, G.; Rarig, R.; Dumitru; O'Connor, C. J. 2006. "Annealing effects on the crystallite size and dielectric properties of ultrafine $\mathrm{Ba} 1-x \operatorname{SrxTiO} 3(0<x<1)$ powders synthesized through an oxalate-complex precursor." Journal of Materials Chemistry 16(8): 752-758.

This Article is brought to you for free and open access by the Department of Chemistry at ScholarWorks@UNO. It has been accepted for inclusion in Chemistry Faculty Publications by an authorized administrator of ScholarWorks@UNO.For more information, please contact scholarworks@uno.edu. 


\title{
Annealing effects on the crystallite size and dielectric properties of ultrafine $\mathrm{Ba}_{1-x} \mathrm{Sr}_{x} \mathrm{TiO}_{3}(0<x<1)$ powders synthesized through an oxalate-complex precursor
}

\author{
Received 10th May 2005, Accepted 29th November 2005 \\ First published as an Advance Article on the web 12th December 2005 \\ DOI: $10.1039 / b 506578 j$
}

Gabriel Caruntu,* Randy Rarig Jr, Ioan Dumitru and Charles J. O'Connor*

\begin{abstract}
Nanocrystalline $\mathrm{Ba}_{1-x} \mathrm{Sr}_{x} \mathrm{TiO}_{3}(0<x<1)$ nanopowders were prepared through the oxalate route and the influence of the thermal history on their dielectric properties was investigated. For pelletized samples obtained by decomposing the corresponding oxalate intermediates at $600{ }^{\circ} \mathrm{C}$ and then annealed at different temperatures ranging from 600 to $1000{ }^{\circ} \mathrm{C}$ the dielectric constant decreases upon increasing the strontium content of the samples. Similar behavior was observed with decreasing the temperature of the thermal treatment of the pellets, a trend which is presumably ascribed to a synergic effect of decreasing the porosity of the samples, the increase of the crystallite size as well as the growth of nanograins. Additionally, a two-fold increase of the dielectric constant is also achievable by using a different annealing strategy, which consists of the direct heat treatment of the intermediates at different temperatures ranging between 600 and $1000{ }^{\circ} \mathrm{C}$. In this latter case, the experimental data unambiguously show a direct correlation between the variation of the dielectric constant and that of the crystallite size/microstrain of the BSTO nanopowders.
\end{abstract}

\section{Introduction}

Alkali-earth titanium oxides $\mathrm{ATiO}_{3}(\mathrm{~A}=\mathrm{Sr}, \mathrm{Ba})$ are important dielectric materials which have been extensively investigated in the last decade, because of their facile synthesis, chemical stability and tunable physical properties. With the continuous demand for miniaturization of multilayered ceramic capacitors, electro-optic devices and dynamic random access (DRAM) ferroelectric memories, the integration of $\mathrm{BaTiO}_{3}$-based devices into the new generation of electronic components represents a high priority. In the last decade, solution-based approaches have become popular for the preparation of nanocrystalline $\mathrm{BaTiO}_{3}$ (BTO) and its strontiumdoped derivatives (BSTO) because of their major advantages over the conventional solid state reactions, namely the high homogeneity and easy control of the particle size/chemical composition of the resulting nanopowders, as well as much lower synthesis temperatures. Thus, $\mathrm{BaTiO}_{3}$ nanoparticles were synthesized by various aqueous approaches, such as sol-gel, ${ }^{1-4}$ hydrothermal, ${ }^{5-9}$ microwave heating, ${ }^{10-12}$ coprecipitation, ${ }^{13,14}$ microemulsion ${ }^{15}$ and thermal decomposition of mixed oxalates, ${ }^{16,17}$ peroxides $^{18-20}$ and citrates. ${ }^{21,22}$ However, the use of aqueous-based chemistry for the synthesis of nanocrystalline $\mathrm{BaTiO}_{3}$ and its strontium-doped derivatives is often hampered by the limited availability of water-soluble $\mathrm{Ti}$ precursors as well as the high sensitivity of titanium precursors towards hydrolysis. Consequently, alternative non-aqueous approaches based on the decomposition of organic barium-titanium precursor complexes, ${ }^{23,24}$ polyol $^{25}$

Advanced Materials Research Institute, University of New Orleans, New Orleans, LA 70148, USA and solvothermal ${ }^{26}$ syntheses have been proposed for the preparation of high quality BTO and BSTO fine powders. Although the non-aqueous approaches are convenient in terms of availability of suitable titanium sources, lower temperature synthesis, size control and degree of dispersion of the resulting nanopowders, the organic precursors are often expensive, the reproducibility of these reactions is often questionable and their scalability is rather limited. Furthermore, extensive research on the dielectric characteristics of $\mathrm{BaTiO}_{3}$ nanopowders have shown that they depend on the synergistic effect of various factors, both synthetic and microstructural, including the chemical composition, the agglomeration degree, the surface area, particle/grain size as well as the porosity of the pelletized samples. Among the various structural parameters which control the dielectric properties of BSTO nanopowders the particle (grain) size is crucial. As grain size is reduced, there is a decrease in the tetragonal lattice ratio constant $(c / a)$ for room temperature crystal structures ${ }^{27-29}$ which causes the main issue with categorizing this effect to lie principally in the synthetic methods. Different synthetic techniques and calcination temperatures influence the grain growth, resulting in widely varying "critical sizes" of $\mathrm{BaTiO}_{3}$. However, while optimization of the values of the dielectric constant can be achieved by rigorous control of the chemical composition and the particle size, only a few papers report on the influence of the crystallite size on the dielectric properties of nanostructured BSTO powders. This work aims at investigating the effect of the annealing temperature and the grain size on the dielectric properties of fine $\mathrm{Ba}_{1-x} \mathrm{Sr}_{x} \mathrm{TiO}_{3}(0<x<1)$ nanopowders obtained by a simple method, that is the thermal decomposition of barium-strontium-titanyl oxalate intermediates at moderate temperatures. 


\section{Experimental}

BSTO nanopowders were prepared by the thermal decomposition of the corresponding barium (strontium)titanyl oxalate complexes. The complex intermediates $\mathrm{Ba}_{1-x} \mathrm{Sr}_{x} \mathrm{TiO}\left(\mathrm{C}_{2} \mathrm{O}_{4}\right)_{2}(0<x<1)$ were synthesized according to a procedure similar to that proposed by Lercher and coworkers. ${ }^{30}$ Reagents used in the preparation of the BSTO nanopowders were reagent grade purity $\mathrm{Ba}\left(\mathrm{NO}_{3}\right)_{2}$ (Alfa Aesar, 99.99\%), $\mathrm{Sr}\left(\mathrm{NO}_{3}\right)_{2}$ (Alfa Aesar, 99.99\%), anhydrous $\mathrm{H}_{2} \mathrm{C}_{2} \mathrm{O}_{4}$ (Alfa Aesar, 98\%) and Ti[O $\left.\left(\mathrm{CH}_{2}\right)_{3} \mathrm{CH}_{3}\right]_{4}$ (Alfa Aesar, 99.9\%) respectively. According to the chart presented in Scheme 1, a stoichiometric amount of $\mathrm{Ti}\left[\mathrm{O}\left(\mathrm{CH}_{2}\right)_{3} \mathrm{CH}_{3}\right]_{4}$ was mixed dropwise with a $10^{-2} \mathrm{M}$ aqueous solution of oxalic acid. A translucent precipitate of $\mathrm{Ti}(\mathrm{OH})_{4}$ resulting from the hydrolysis of $\mathrm{TiO}^{2+}$ ions formed immediately, but disappeared within 30 minutes under continuous stirring at room temperature and finally led to a yellowish solution of titanyl oxalate.

Then, different aqueous solutions containing a stoichiometric amount of $\mathrm{Ba}\left(\mathrm{NO}_{3}\right)_{2}, \operatorname{Sr}\left(\mathrm{NO}_{3}\right)_{2}$ or a mixture of $\mathrm{Ba}\left(\mathrm{NO}_{3}\right)_{2}-\mathrm{Sr}\left(\mathrm{NO}_{3}\right)_{2}$, according to the desired $\mathrm{Ba} / \mathrm{Sr}$ ratio, were prepared separately and poured into the titanyl oxalate solution, with instantaneous formation of a white $\mathrm{Ba}_{1-x} \mathrm{Sr}_{x} \mathrm{TiO}\left(\mathrm{C}_{2} \mathrm{O}_{4}\right)_{2} \cdot x \mathrm{H}_{2} \mathrm{O}$ intermediate. The white powders were further separated by filtration, washed several times with deionized water and dried overnight at room temperature under vacuum. Identification of the crystalline phases and the phase purity of the nanopowders were performed by X-ray diffraction using a Philips X'Pert Pro MPD system equipped

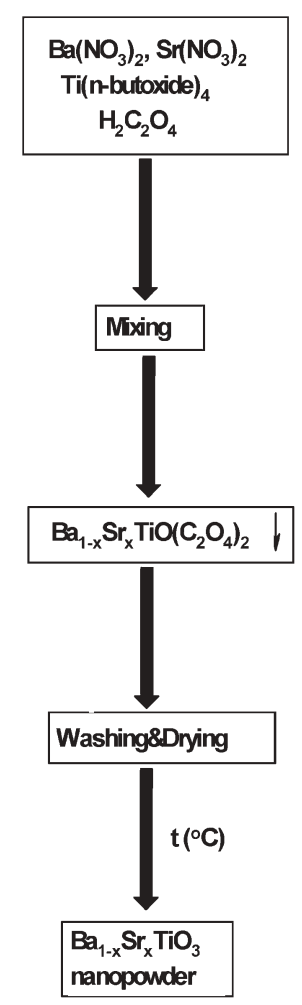

Scheme 1 Synthetic methodology for the preparation $\mathrm{Ba}_{1-x} \mathrm{Sr}_{x} \mathrm{TiO}_{3}$ nanopowders via thermal decomposition $\mathrm{Ba}_{1-x} \mathrm{Sr}_{x} \mathrm{TiO}\left(\mathrm{C}_{2} \mathrm{O}_{4}\right)_{2}$ intermediate. with a curved graphite single-crystal monochromator $\mathrm{Cu}$ $\mathrm{K} \alpha$ radiation). Line profile analysis and estimation of the crystallite size were performed by using the Williamson-Hall methodology $y^{31}$ from the corresponding XRD patterns recorded in a step scanning mode in the $20-95^{\circ} 2 \theta$ range with a step of $0.02^{\circ}$ and a counting time of $10 \mathrm{~s}$. The morphology of the nanopowders and their chemical composition were studied by using a JEOL-JSM 5410 scanning electron microscope (SEM), whereas the transmission electron microscopy (TEM) was performed with a JEOL 2010, Supertwin instrument, with point-to-point resolution of $0.23 \mathrm{~nm}$ and a line resolution of $0.14 \mathrm{~nm}$. For TEM examination, the colloidal BSTO powders were dispersed in absolute ethanol, sonicated for $15 \mathrm{~min}$ and then placed onto carbon coated TEM grids. Investigation of the dielectric properties of the BSTO nanopowders was performed on nanopowders pressed into pellets with an Agilent HP4284A precision LCR analyzer working in the frequency range of $20 \mathrm{~Hz}-1 \mathrm{MHz}$ with a $10^{-3} \mathrm{~Hz}$ resolution.

\section{Results and discussion}

As presented in Scheme 2, two different approaches were used to ascertain the role of the annealing effects on the dielectric properties of the BSTO nanopowders obtained by the thermal decomposition of the mixed oxalate intermediates. Consequently, the results and discussion will be divided into two parts: the first section will detail the structure, morphology and the dielectric properties of the $\mathrm{Ba}_{1-x} \mathrm{Sr}_{x} \mathrm{TiO}_{3}(0<x<1)$ (BSTO) nanopowders obtained by the decomposition of the barium-strontium-titanyl oxalates at $600{ }^{\circ} \mathrm{C}$ (denoted by 1 in Scheme 2). To measure their dielectric properties, the samples were pelletized and subjected to a supplementary annealing at different temperatures ranging from $600{ }^{\circ} \mathrm{C}$ to $1000{ }^{\circ} \mathrm{C}$, thereby reducing their porosity level and enhancing the intimate contact between the nanograins.

The second series of nanocrystalline BSTO samples (denoted by 2 in Scheme 2) was obtained by the direct thermal

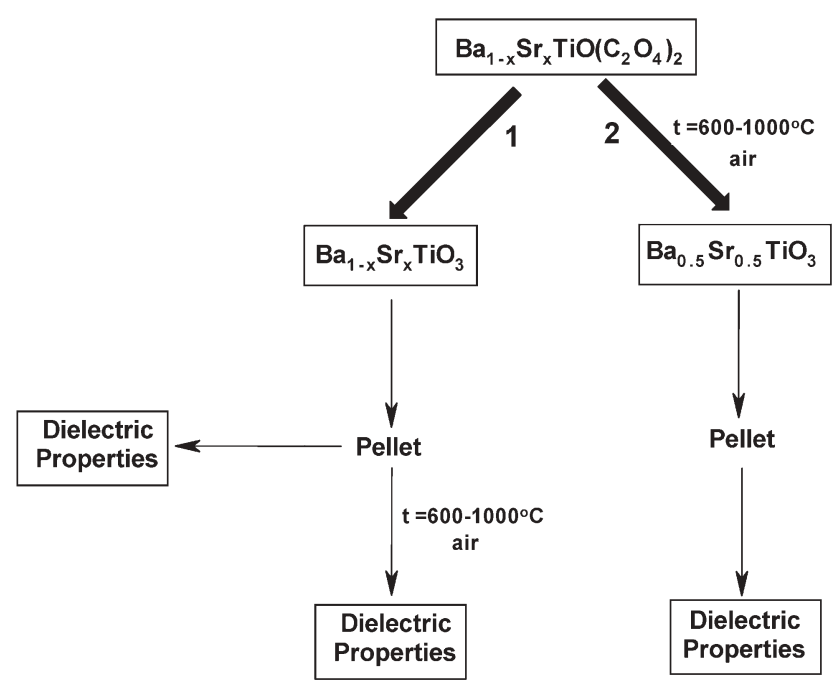

Scheme 2 Experimental procedure for the preparation of different pelletized samples subjected to the measurement of dielectric properties. 
decomposition of the corresponding intermediates at different temperatures, ranging from $600{ }^{\circ} \mathrm{C}$ to $1000{ }^{\circ} \mathrm{C}$. Prior to measurement of their dielectric properties, the obtained products were pelletized and subjected to a short annealing in air at $600{ }^{\circ} \mathrm{C}$ for $30 \mathrm{~min}$, thus allowing the densification of the pellets and the monitoring of the influence of the crystallite size on their dielectric properties. Unless otherwise stated, the annealing time was for all series of samples $6 \mathrm{~h}$ in open atmosphere. Although the investigation of the dielectric properties was performed for the whole compositional range $0<x<1$ ( $x=0$; $0.2 ; 0.4 ; 0.5 ; 0.6 ; 0.8$ and 1 ), for the sake of brevity the discussion will be focused only on $\mathrm{Ba}_{0.5} \mathrm{Sr}_{0.5} \mathrm{TiO}_{3}$.

For the first series of samples, the structural characteristics of the BSTO nanopowders were found to be similar regardless of the strontium composition and are exemplified by $\mathrm{Ba}_{0.5} \mathrm{Sr}_{0.5} \mathrm{TiO}_{3}$, which was chosen as a model system.

According to the corresponding XRD pattern, the cubic $\mathrm{Ba}_{0.5} \mathrm{Sr}_{0.5} \mathrm{TiO}_{3}$ powders are single phase and highly crystalline (Fig. 1). Additionally, the refined value of the lattice parameter is $a=3.97(1) \AA$, which is close to the value of $a=3.94 \AA$, reported for bulk $\mathrm{BaTiO}_{3}{ }^{32}$

Furthermore, careful examination of the (200) peak located at $2 \theta \approx 45^{\circ}$ (inset of Fig. 1) reveals its shape symmetry and the absence of splitting, which are indicative of a cubic symmetry of the BaSTO nanopowders. Although our discussion was limited to $\mathrm{BaTiO}_{3}$, it is worth mentioning that for all compositions $\mathrm{Ba}_{1-x} \mathrm{Sr}_{x} \mathrm{TiO}_{3}(0<x<1)$ obtained by annealing of the $\mathrm{Ba}_{1-x} \mathrm{Sr}_{x} \mathrm{TiO}\left(\mathrm{C}_{2} \mathrm{O}_{4}\right)_{2}$ intermediates, broadening of the (200) reflection was not observed. This result is in agreement with the general observation that the tetragonal distortion becomes less pronounced in fine $\mathrm{BaTiO}_{3}$ powders as the particle size decreases and the Curie temperature $T_{\mathrm{c}}$ decreases accordingly to approach room temperature. ${ }^{33-34}$ However, the "critical size", which corresponds to the cubic-tetragonal phase transition in BSTO nanopowders originates mainly from the strain between the grains which, in turn, is strongly dependent on the preparation method.

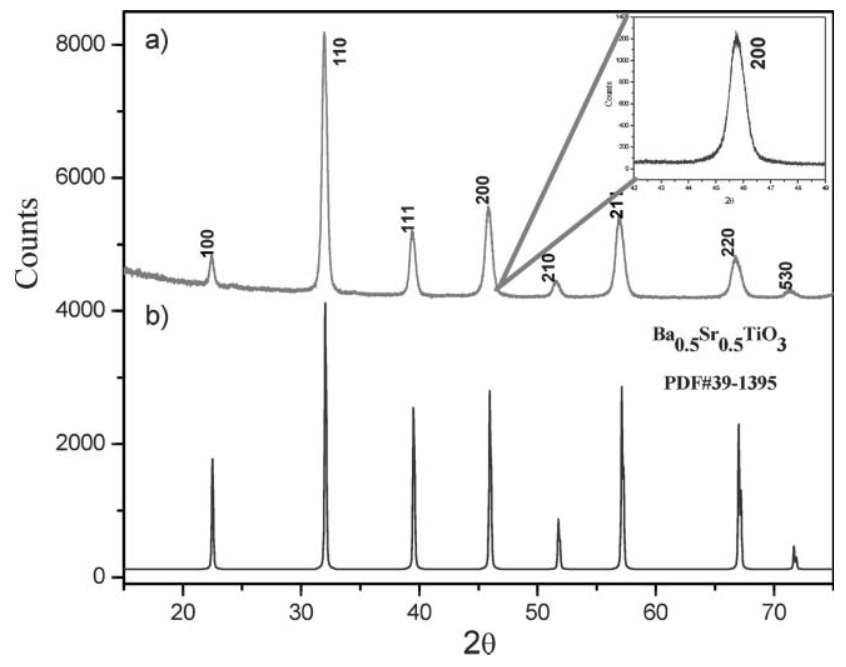

Fig. 1 Typical XRD pattern of nanocrystalline $\mathrm{BaTiO}_{3}$ obtained by annealing of the $\mathrm{BaTiO}\left(\mathrm{C}_{2} \mathrm{O}_{4}\right)_{2}$ intermediate at $600{ }^{\circ} \mathrm{C}$ in air (a) and the simulated XRD pattern for the bulk $\mathrm{BaTiO}_{3}(\mathrm{~b})$.
Electron microscopy was used to investigate the particle/ grain size and morphology of the $\mathrm{Ba}_{1-x} \mathrm{Sr}_{x} \mathrm{TiO}_{3}$ nanopowders obtained by the thermal decomposition of the bariumstrontium-titanyl oxalate precursors at $600{ }^{\circ} \mathrm{C}$. Examination of the TEM micrograph of the nanocrystalline $\mathrm{Ba}_{0.5} \mathrm{Sr}_{0.5} \mathrm{TiO}_{3}$, Fig. 2(a), reveals that the sample is composed of agglomerated spherical nanoparticles with an estimated average diameter of $120 \mathrm{~nm}$.

Moreover, the selected area electron diffraction pattern (SAED) reveals that individual nanoparticles are single crystalline in nature, possessing a cubic symmetry, in agreement with the results obtained from the X-ray diffraction study (Fig. 2b). The SEM picture shown in Fig. 2(c) reveals that at the microscale, the agglomerated BSTO nanopowders are composed of well defined spherical, highly uniform grains, with a variable diameter of $1-2 \mu \mathrm{m}$ possessing irregular surfaces (inset of Fig. 2(c)).

As previously mentioned, in order to measure their dielectric properties the first series of BSTO nanopowders were pelletized ( $\sim 48500 \mathrm{psi}$ ) and subjected to a second annealing at different temperatures, ranging between $600-1000{ }^{\circ} \mathrm{C}$, in open air. Fig. 3 shows the variation of the dielectric properties of the as-pelletized BSTO samples as a function of the frequency and the strontium content. Thus, two important trends are observed: (i) the dielectric constant and the loss tangent present their highest values at low frequencies $(f=$ $20 \mathrm{~Hz}$ ) and then decrease slowly with increasing the frequency (Fig. 3a and b) and (ii) the dielectric constant decreases monotonically upon the increasing of the strontium content (Fig. 3c). For a constant frequency, the values of dielectric constant of the corresponding as-pelletized BSTO samples were found to decrease monotonically with increasing the strontium content from $\varepsilon=97$ for $\mathrm{BaTiO}_{3}$ to $\varepsilon=44$ for $\mathrm{SrTiO}_{3}$.

A similar trend was observed in the case of the loss tangent, that is, it decreases from a value of 0.5 at $20 \mathrm{~Hz}$ to a value of $2 \times 10^{-2}$ at frequencies above $200 \mathrm{kHz}$. However, the values of the dielectric constant measured for the as-pelletized sample are much lower than the values reported for the corresponding bulk materials, which is presumably due to the high degree of

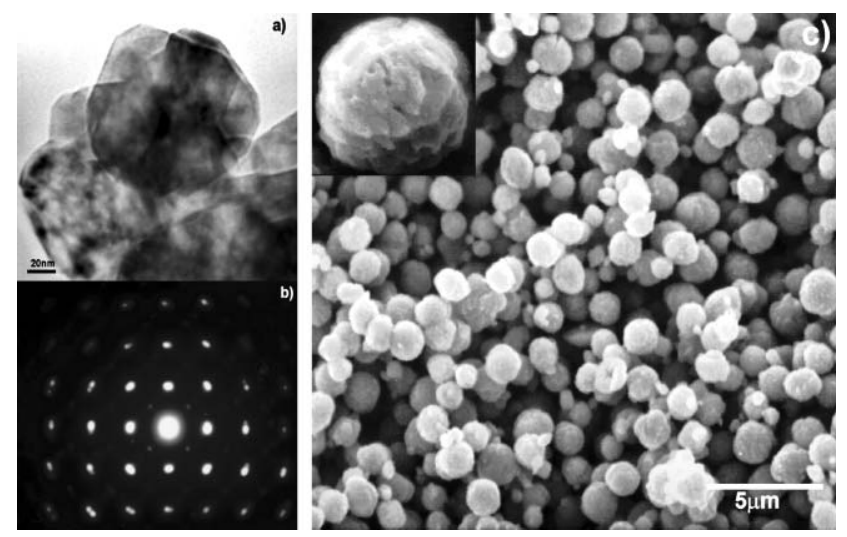

Fig. 2 Typical TEM and SEM micrographs of $\mathrm{Ba}_{0.5} \mathrm{Sr}_{0.5} \mathrm{TiO}_{3}$ nanopowders obtained by annealing of the $\mathrm{BaTiO}\left(\mathrm{C}_{2} \mathrm{O}_{4}\right)_{2}$ intermediate at $600{ }^{\circ} \mathrm{C}$ in air: (a) TEM image of nanocrystalline $\mathrm{BaTiO}_{3}$, (b) selected area electron diffraction pattern of a single $\mathrm{Ba}_{0.5} \mathrm{Sr}_{0.5} \mathrm{TiO}_{3}$ grain, (c) top-down SEM image of nanocrystalline $\mathrm{Ba}_{0.5} \mathrm{Sr}_{0.5} \mathrm{TiO}_{3}$. 

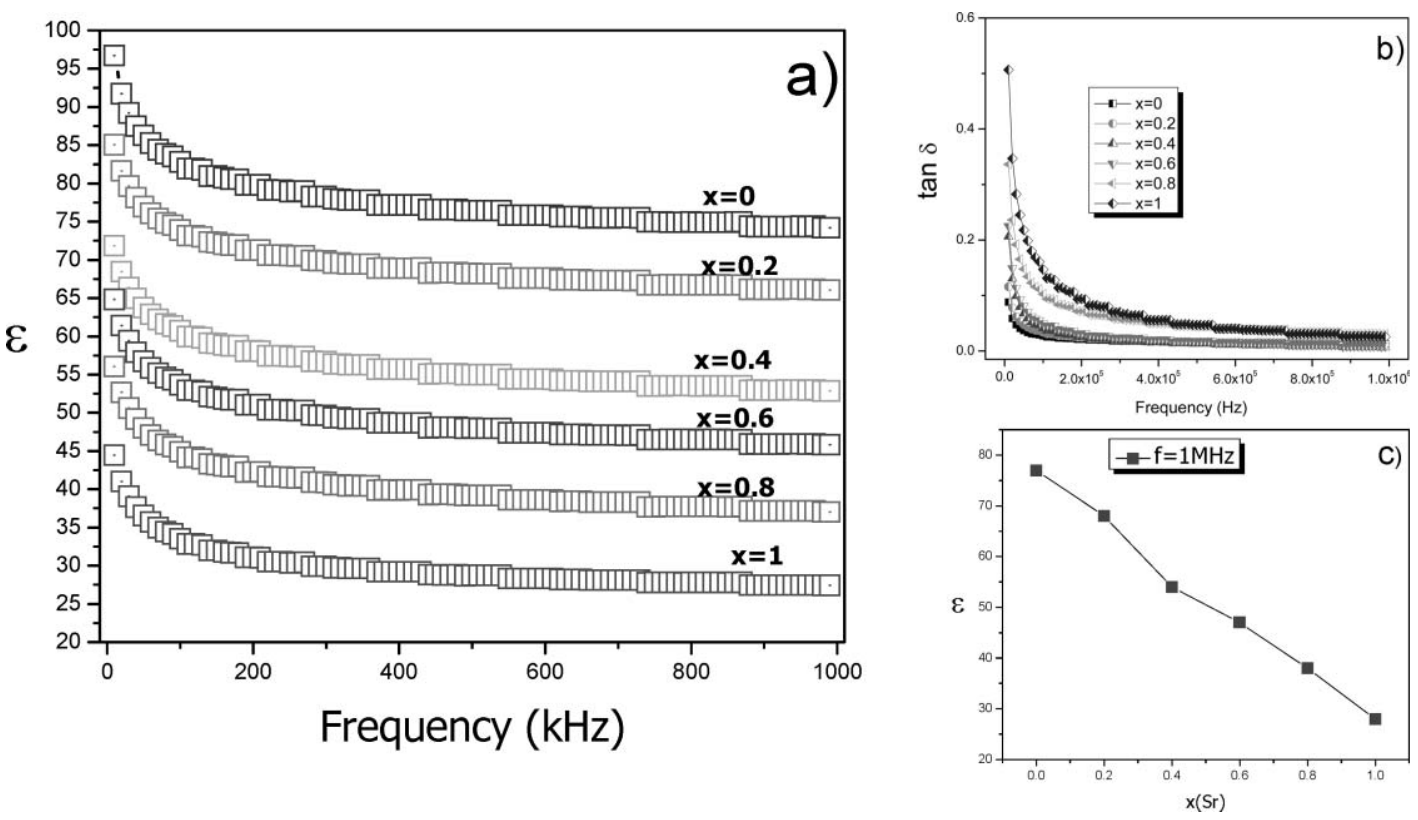

Fig. 3 Variation of the dielectric constant (a) and loss tangent (b) of $\mathrm{Ba}_{1-x} \mathrm{Sr}_{x} \mathrm{TiO}_{3}$ nanopowders $(0<x<1)$ with the frequency. The BSTO fine powders were obtained by thermal decomposition of the complex oxalate precursor at $600{ }^{\circ} \mathrm{C}$ in air and the products were pelletized into discs. (c) Variation of the dielectric constant of the samples with the strontium content for samples measured at $f=1 \mathrm{MHz}$.

porosity of pelletized samples not subjected to a supplementary annealing. However, it is well known that the dielectric properties of BSTO powders can be considerably enhanced upon thermal treatment principally because of their densification and evolution of the grain boundaries along with the modification of the domain structure and the topography of the crystal defects. ${ }^{35,36}$ To confirm such a trend, the pelletized samples were subjected to a supplementary annealing at temperatures ranging between 600 and $1000{ }^{\circ} \mathrm{C}$ for $6 \mathrm{~h}$ in air. The dependence of the dielectric constant measured at $20 \mathrm{kHz}$ on the sintering temperature of these pellets is presented in Fig. 4.

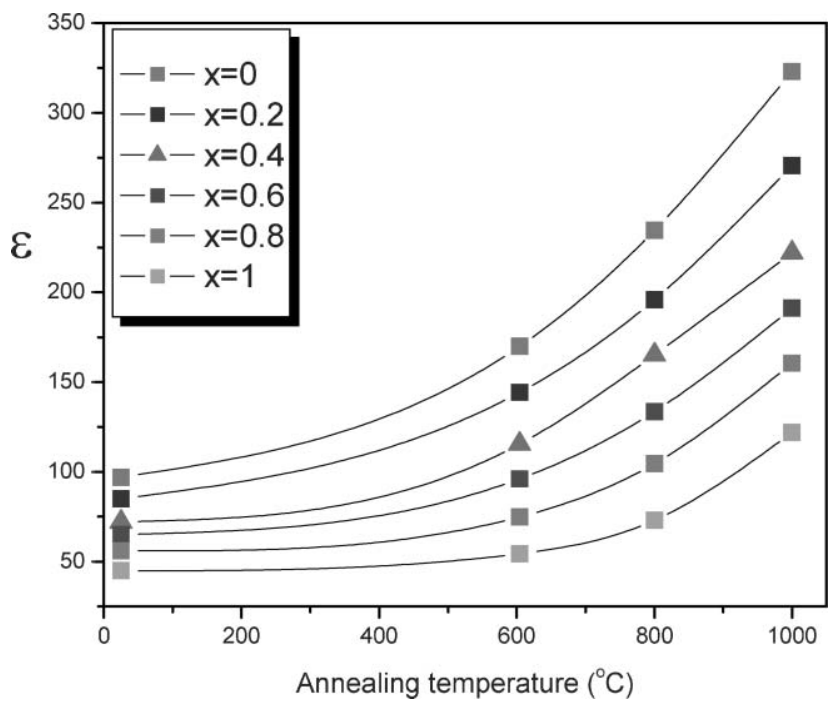

Fig. 4 Evolution of the dielectric constant with the strontium content and sintering temperature for the $\mathrm{Ba}_{1-x} \mathrm{Sr}_{x} \mathrm{TiO}_{3}$ nanopowders obtained by the thermal decomposition of the oxalate complex at $600{ }^{\circ} \mathrm{C}$.
As seen in Fig. 4, the dielectric constant of the sintered pellets increases almost three times with increasing of the annealing temperature. Thus, the dielectric constant of the $\mathrm{BaTiO}_{3}$ nanopowders $(x=0)$ measured at $20 \mathrm{~Hz}$ increases slightly from $\varepsilon=97$ for the as-pelletized sample to $\varepsilon_{600}=171$ for the pellet annealed at $600{ }^{\circ} \mathrm{C}$, and reaches a maximum of $\varepsilon=313$ for the pellets annealed at $1000{ }^{\circ} \mathrm{C}$. The increase of the dielectric constant with increasing the annealing temperature of the pellets is mainly due to the densification of the samples and the reduction of their porosity. It is worth noting that the strontium content clearly influences the dielectric constant of the nanocrystalline BSTO sample. Consequently, the highest values of the dielectric constant are observed in the case of the samples with the lowest strontium content $(x=0$ and $x=0.2)$ after annealing at $1000{ }^{\circ} \mathrm{C}$. In this case the dielectric constant is almost six times higher than those measured in the case of the pelletized samples which were not subjected to a heat treatment. The samples with an intermediate strontium content ( $x=0.4-0.6)$ show a smaller increase of the dielectric constant with the annealing temperature, where the dielectric constant for the pellet annealed at $1000{ }^{\circ} \mathrm{C}$ is almost three times higher than that of the as-prepared pellet.

Finally, for the pellets with the richest strontium content $(x=0.8-1.0)$ the dielectric constant shows a much lower increase with increasing annealing temperature. However, the measured values of the dielectric constant $(\varepsilon=310)$ are lower than the values reported by Ganguli and coworkers ${ }^{21}$ for BSTO pellets sintered at $1100{ }^{\circ} \mathrm{C}\left(\varepsilon_{\mathrm{BaTiO} 3}=510\right.$ and $\varepsilon_{\mathrm{SrTiO} 3}=$ 170 ), which can be ascribed to the lower sintering temperature used in our experiments $\left(1000{ }^{\circ} \mathrm{C}\right.$ versus $\left.1100{ }^{\circ} \mathrm{C}\right)$, as well as the different synthetic method. In the case of the second series of samples, the oxalate intermediate was heat treated at different temperatures, typically ranging between 600 and $1000{ }^{\circ} \mathrm{C}$, then pelletized, annealed at $600{ }^{\circ} \mathrm{C}$ for 30 minutes to reduce their 


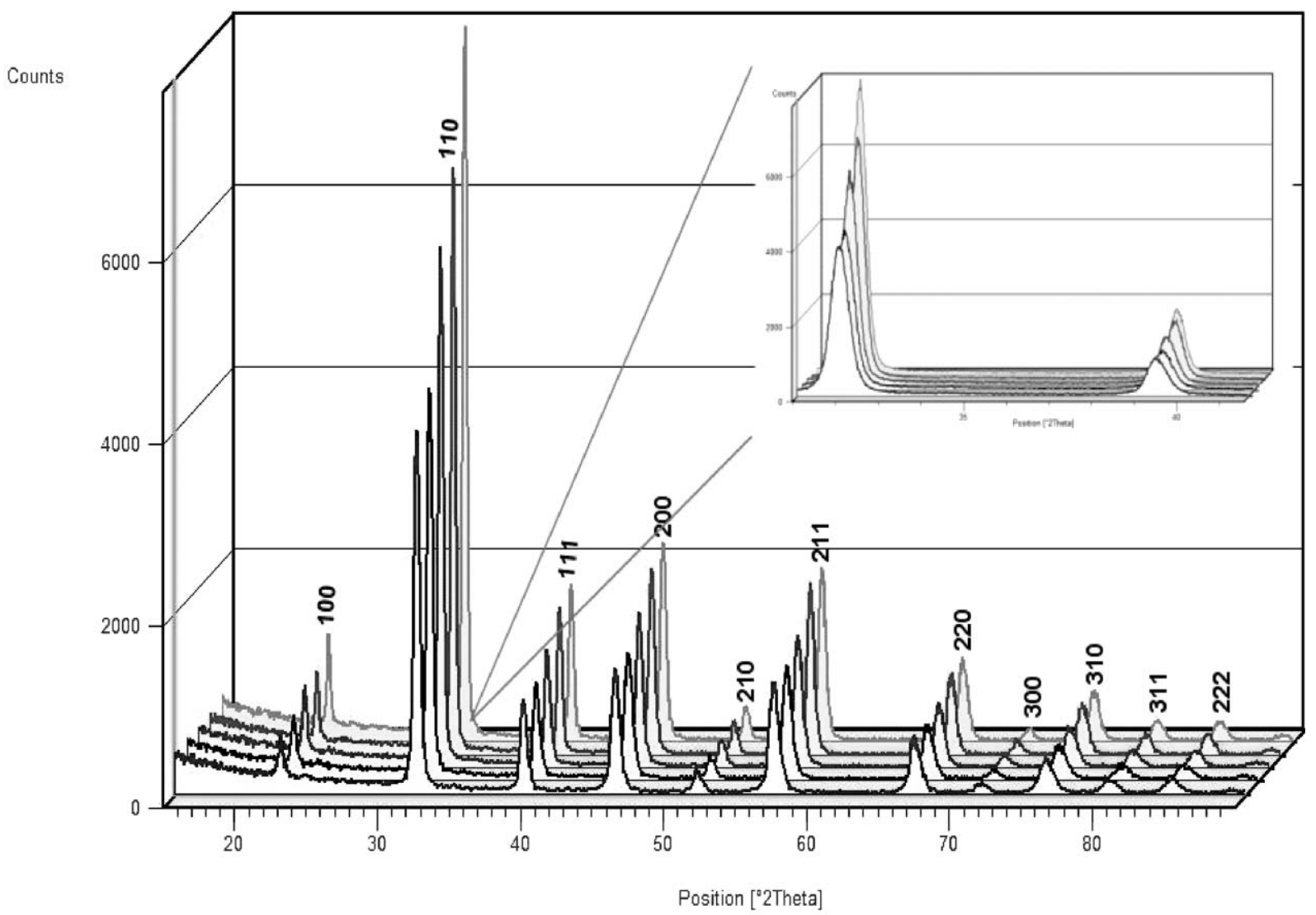

Fig. 5 X-Ray diffraction patterns of $\mathrm{Ba}_{0.5} \mathrm{Sr}_{0.5} \mathrm{TiO}_{3}$ nanopowders obtained by the heat treatment of the oxalate intermediate at different temperatures (from $600{ }^{\circ} \mathrm{C}$ for the front pattern to $1000{ }^{\circ} \mathrm{C}$ for the far back pattern in $200{ }^{\circ} \mathrm{C}$ increments). The inset represents a close-up of the (220) peak, showing the absence of splitting.

porosity and their dielectric properties measured. Fig. 5 is a 3D plot of the X-ray diffraction patterns of the products obtained by the heat treatment of the oxalate intermediates. Similar to the first series of samples, it can be seen that the annealing process leads to single, highly crystalline phases which can be indexed into a cubic symmetry. The inset of the picture is a close-up of the (200) reflection which also presents no splitting and becomes narrower when increasing the annealing temperature.

The evolution of the crystallite size with the annealing temperature was examined from the corresponding X-ray diffraction pattern for the sample having the chemical composition of $\mathrm{B}_{\mathrm{a} 0.5} \mathrm{Sr}_{0.5} \mathrm{TiO}_{3}$ by using the peak-profile analysis. Correspondingly, the Williamson-Hall plot was used to estimate the crystallite size and the strain of the crystallites:

$$
B \cos \theta=\frac{0.9 \lambda}{t}+2 \frac{\Delta d}{d} \sin \theta
$$

where $B$ represents the full-width at half maximum (FWHM) of the peak appearing in the XRD pattern at the angle $2 \theta, \lambda$ is the wavelength (for $\mathrm{Cu} \mathrm{K} \alpha$ radiation $\lambda=154056 \AA$ ), $\Delta d l d$ the strain and $t$ the crystallite size. Fig. 6 details the experimental results obtained by using the Williamson-Hall methodology for the $\mathrm{Ba}_{0.5} \mathrm{Sr}_{0.5} \mathrm{TiO}_{3}$ nanopowders obtained by annealing of the oxalate complex at different temperatures for $6 \mathrm{~h}$ in air. In Fig. 6(a) is shown the variation of the crystallite size and strain as obtained from the linear interpolation of eqn (1) as a function of the annealing temperature (Fig. 6b).

Fig. 6a clearly shows that the crystallite size increases uniformly from 32 to $94 \mathrm{~nm}$ with increasing the sintering temperature of the complex intermediate. Similarly, the strain $\Delta d l d$ follows the opposite trend, that is, it decreases from 6000 to $\sim 3500$. These variations can be assigned to the change in the microstructure of the nanostructured powders during the thermal treatment. As previously shown, during the heat treatment of the carboxylate precursors, they decompose in the temperature range of $460-530{ }^{\circ} \mathrm{C}$. Additionally, from the linear dependence of the dielectric constant of the BSTO nanopowders as a function of the crystallite size (Fig. 6c) it becomes clear that the dielectric properties of these nanostructured BSTO powders can be finely tuned through the rigorous control of some of their key structural parameters such as the crystallite size. In Fig. 7 is represented the frequency variation of the dielectric constant of the pelletized $\mathrm{Ba}_{0.5} \mathrm{Sr}_{0.5} \mathrm{TiO}_{3}$ nanopowders. As mentioned before, these samples were obtained by thermal decomposition of the corresponding mixed oxalate intermediates and their dielectric properties were measured at $20 \mathrm{~Hz}$.

As in the case of the first series of samples, the initial values of the dielectric constant present a maximum at low frequencies which decreases gradually with increasing the frequency up to $1 \mathrm{MHz}$. As for the variation of the dielectric 
(a)

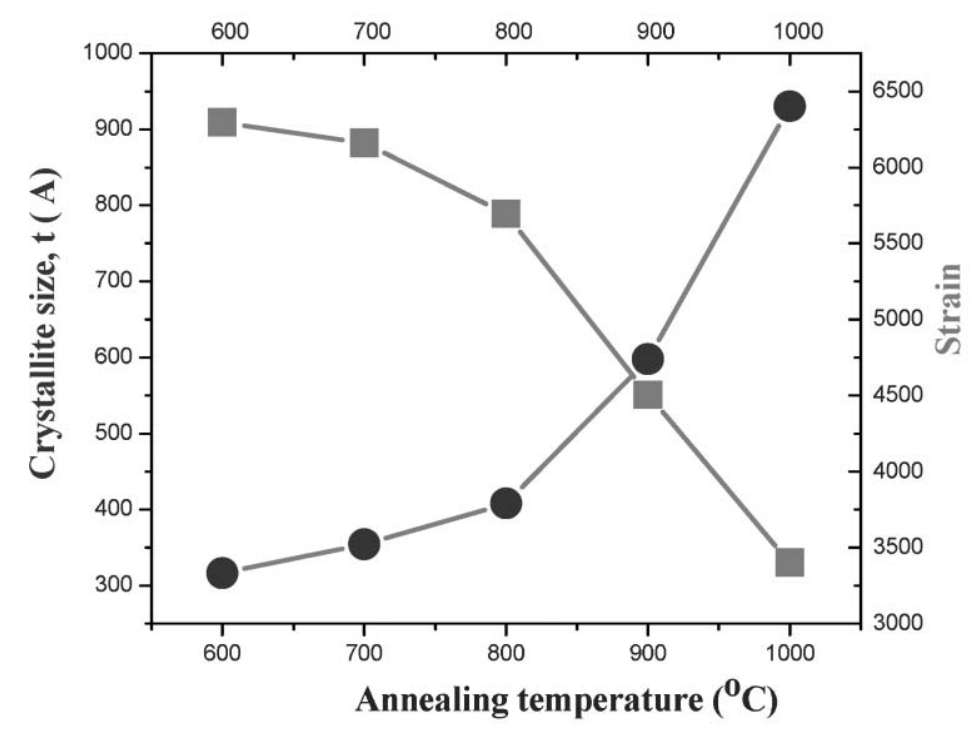

(b)

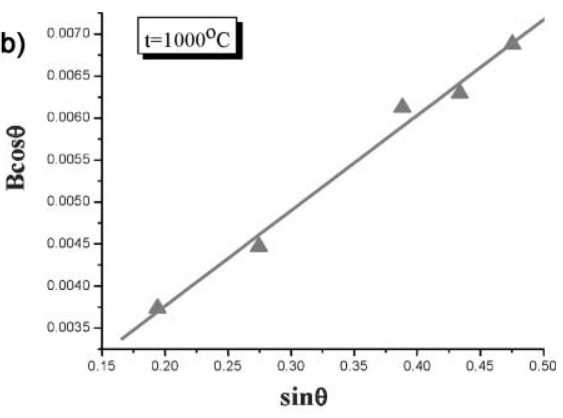

(c)

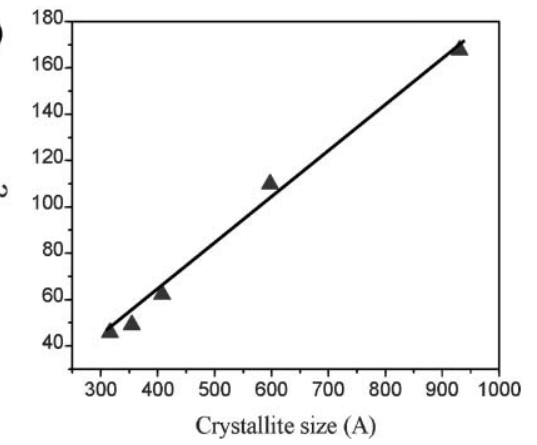

Fig. 6 (a) Variation of the crystallite size (circles) and strain (squares) with the annealing temperature of $\mathrm{Ba}_{0.5} \mathrm{Sr}_{0.5} \mathrm{TiO}_{3}$ nanopowders; (b) a representative Williamson-Hall plot; (c) variation of the dielectric constant (measured at $1 \mathrm{MHz}$ ) as a function of the crystallite size.

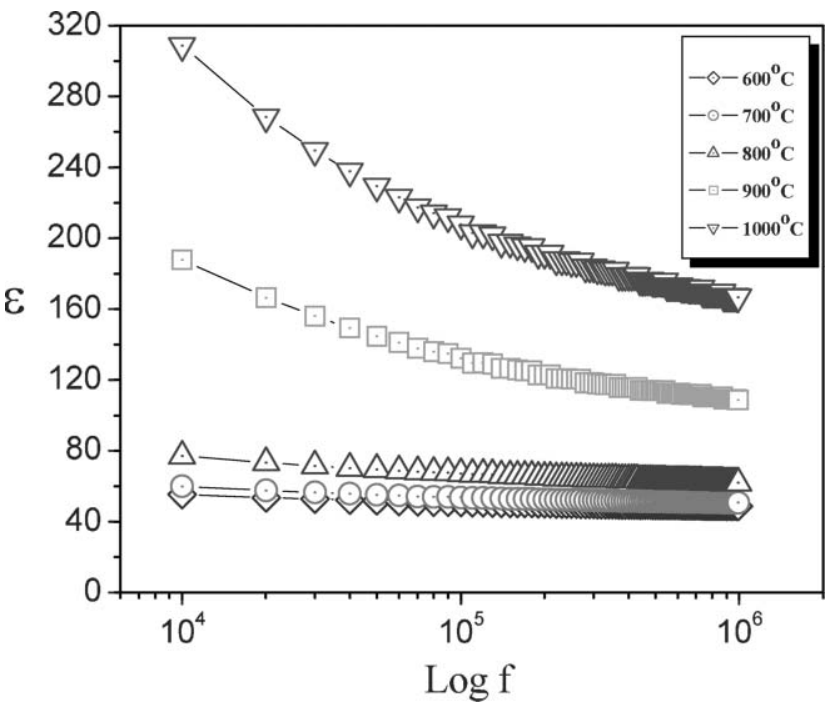

Fig. 7 Frequency dependence of the dielectric constant $\varepsilon$ for the $\mathrm{Ba}_{0.5} \mathrm{~S}_{\mathrm{r} 0.5} \mathrm{TiO}_{3}$ nanopowders obtained by the thermal decomposition of the oxalate complex at different temperatures.

constant with the annealing temperatures, between 600 and $800{ }^{\circ} \mathrm{C}$ the increase is small whereas for higher annealing temperatures this increase is observed to a greater extent. Again, the dielectric properties of the $\mathrm{Ba}_{0.5} \mathrm{Sr}_{0.5} \mathrm{TiO}_{3}$ samples were found to depend strongly on their thermal history, that is the dielectric constant increases with increasing the decomposition temperature of the intermediate complex. A comparison of the dielectric properties of BSTO samples obtained by the two different annealing strategies indicates that the dielectric constants of the BSTO samples obtained by direct thermal decomposition of the complex intermediates at temperatures ranging between $600-1000{ }^{\circ} \mathrm{C}$ are much higher than those of the samples obtained from the oxalate complex at $600{ }^{\circ} \mathrm{C}$ and then annealed at different temperatures in the same temperature range. As an example, the dielectric constant measured at $20 \mathrm{~Hz}$ for the $\mathrm{Ba}_{0.5} \mathrm{Sr}_{0.5} \mathrm{TiO}_{3}$ nanopowders obtained by the first annealing strategy after annealing at $1000{ }^{\circ} \mathrm{C}$ was about 190 , whereas that measured for the sample obtained following the second pathway is 320 . Accordingly, we believe that the direct annealing of the complex intermediate without a subsequent heat treatment reduces the porosity of the BSTO nanopowders to a greater extent, thus enhancing their dielectric properties.

\section{Conclusions}

In summary, nanostructured BSTO powders with variable composition were prepared by thermal decomposition of the corresponding oxalate complex intermediates and the influence of the thermal history on their dielectric properties was investigated. Subsequently, the annealing effects on the crystallite size and dielectric properties of the resulted nanopowders are described. When the powders were obtained by decomposition of the oxalate complex at $600{ }^{\circ} \mathrm{C}$ and then subjected to a supplementary heat treatment at temperatures ranging between 600 and $1000{ }^{\circ} \mathrm{C}$ the above results show that the dielectric constant increases with decreasing the strontium composition and with increasing the annealing temperature. The same trend was observed for BSTO samples obtained by a different annealing pathway; that is by directly heating the oxalate complex intermediate at different temperatures ranging between 600 and $1000{ }^{\circ} \mathrm{C}$. However, in this latter case, 
as exemplified for $\mathrm{Ba}_{0.5} \mathrm{Sr}_{0.5} \mathrm{TiO}_{3}$ nanopowders, the dielectric constant is almost two-fold greater than that of the powders obtained by the two sequential heat treatments. Such a variation of the dielectric properties is conceivably due to major changes in the microstructure of the powders during the heat treatments, that is the increase of the grain size, as well as the reduction of the porosity and the growth and consolidation of the nanograins.

\section{Acknowledgements}

This work was supported by DOD/DARPA through Grant No. MDA 972-97-1-0003.

\section{References}

1 U. Hwang, H. S. Park and K. K. Koo, J. Am. Ceram. Soc., 2004, 87, 12, 2168.

2 D. Demydov and K. J. Klabunde, J. Non-Cryst. Solids, 2004, 350, 165.

3 D. Hreniak, E. Lukowiak, K. Maruszewski, R. Pazik and W. Strek, Mater. Sci., 2002, 20, 1, 43.

4 R. Pazik, D. Hreniak and W. Strek, Mater. Sci., 2004, 22, 3, 219.

5 J. Moon, E. Suvaci, A. Morrone, S. Costantino and J. H. Adair, J. Eur. Ceram. Soc., 2003, 23, 12, 2153.

6 Y. Hakuta, H. Ura, H. Hayashi and K. Arai, Ind. Eng. Chem. Res., $2005,44,4,840$.

7 H. Nakano, K. Urabe, T. Oikawa and H. Ikawa, J. Am. Ceram. Soc., 2004, 87, 8, 1594.

8 M. A. McCormick, R. K. Roeder and E. B. Slamovich, J. Mater. Res., 2001, 16, 4, 1200.

9 B. L. Gersten, M. M. Lencka and R. E. Riman, J. Am. Ceram. Soc., 2004, 87, 11, 2025.

10 Y. S. Malghe, A. V. Gurjar and S. R. Dharwadkar, Bull. Mater. Sci., 2004, 27, 3, 217.

11 Y. Ma, M. Vileno, L. Suib and P. K. Dutta, Chem. Mater., 1997, 9, $12,3023-3031$.

12 B. L. Newalkar, S. Komarneni and H. Katsuki, Mater. Res. Bull., 2001, 36, 13-14, 2347.

13 J. Zhu, S. Wu, H. Cheng, Z. Yin and J. Jeon, Mater. Sci. Forum, 2003, 423-425, 417.
14 H. S. Potdar, S. B. Deshpande and S. K. Date, Mater. Chem. Phys., 1999, 58, 2, 121.

15 H. Herrig and R. Hempelmann, Nanostruct. Mater., 1997, 9, 1-8, 241.

16 W. S. Clabaugh, E. M. Swiggard and R. Gilchrist, J. Res. Natl. Bur. Stand., 1956, 56, 289.

17 S. Gijp, L. Winnubst and H. Verweij, J. Mater. Chem., 1998, 8, 5, 1251.

18 (a) G. Z. Pfaff, J. Eur. Ceram. Soc., 1992, 27, 5, 1222; (b) B. W. Lee and S. B. Cho, J. Electroceram., 2004, 13, 1/2/3, 379.

19 Z. Peng and Y. Chen, Microelectron. Eng., 2003, 66, 1-4, 102.

20 M. Tada, K. Tomita, V. Petrykin and M. Kakihana, Solid State Ionics, 2002, 151, 293.

21 P. R. Arya, P. Jha and A. K. Ganguli, J. Mater. Chem., 2003, 13, 415.

22 L. A. Perez-Maqueda, M. J. Dianez, F. J. Gotor, M. J. Sayagues, C. Real and M. J. Criado, J. Mater. Chem., 2003, 13, 2234.

23 S. O'Brien, L. Brus and C. B. Murray, J. Am. Chem. Soc., 2001, 123, 12085 .

24 J.J. Urban, W. S. Yun, Q. Gu and H. Park, J. Am. Chem. Soc., 2002, 124, 1186.

25 W. E. Rhine and R. B. Hallock, Chem. Mater., 1992, 4, 1208.

26 D. Chen and X. Jiao, J. Am. Ceram. Soc., 2000, 83, 10, 2637-2639.

27 R. Asiaie, W. Zhu, A. Akbar and P. K. Dutta, Chem. Mater., 1996, 8, 226.

28 B. Begg, E. R. Vance and J. Nowotny, J. Am. Ceram. Soc., 1994, 77, 3186.

29 S. Wada, H. Yasuno, T. Hoshina, S. Nam, H. Kakemoto and T. Tsurumi, Ceram. Trans., 2004, 150, 205.

30 M. Stockenhuber, H. Mayer and J. A. Lercher, J. Am. Ceram. Soc., 1993, 76, 5, 1185.

31 G. K. Williamson and W. H. Hall, Acta Metall., 1953, 1, 1, 22.

32 R. Waesche, W. Denner and H. Schultz, Mater. Res. Bull., 1981, 16, 497.

33 Z. Zhao, V. Buscaglia, M. Viviani, M. T. Buscaglia, L. Mitoseriu, A. Testino, M. Nygren, M. Johnsson and P. Nanni, Phys. Rev. B, 2004, 70, 24107.

34 K. Uchino, E. Sadanaga and T. Hirose, J. Am. Ceram. Soc., 1989, 72, 8, 1555-1558.

35 B. Su, J. Y. He, B. L. Cheng, T. W. Button, J. Liu, Z. Shen and M. Nygren, Integr. Ferroelectr., 2004, 61, 117.

36 A. V. Ragulya and V. V. Skorokhod, NATO Sci. Ser., II, 2003, 128, 139 (Nanostructures: Synthesis, Functional Properties and Applications). 